\title{
Papers
}

\section{Quantifying effect of statins on low density lipoprotein cholesterol, ischaemic heart disease, and stroke: systematic review and meta-analysis}

\author{
M R Law, N J Wald, A R Rudnicka
}

\begin{abstract}
Objectives To determine by how much statins reduce serum concentrations of low density lipoprotein (LDL) cholesterol and incidence of ischaemic heart disease (IHD) events and stroke, according to drug, dose, and duration of treatment.

Design Three meta-analyses: 164 short term randomised placebo controlled trials of six statins and LDL cholesterol reduction; 58 randomised trials of cholesterol lowering by any means and IHD events; and nine cohort studies and the same 58 trials on stoke.

Main outcome measures Reductions in LDL cholesterol according to statin and dose; reduction in IHD events and stroke for a specified reduction in LDL cholesterol.

Results Reductions in LDL cholesterol (in the 164 trials) were $2.8 \mathrm{mmol} / \mathrm{l}(60 \%)$ with rosuvastatin 80 $\mathrm{mg} /$ day, $2.6 \mathrm{mmol} / \mathrm{l}(55 \%)$ with atorvastatin 80 $\mathrm{mg} /$ day, $1.8 \mathrm{mmol} / \mathrm{l}(40 \%)$ with atorvastatin 10 $\mathrm{mg}$ /day, lovastatin $40 \mathrm{mg}$ /day, simvastatin $40 \mathrm{mg} /$ day, or rosuvastatin $5 \mathrm{mg} /$ day, all from pretreatment concentrations of $4.8 \mathrm{mmol} / \mathrm{l}$. Pravastatin and fluvastatin achieved smaller reductions. In the 58 trials, for an LDL cholesterol reduction of $1.0 \mathrm{mmol} / \mathrm{l}$ the risk of IHD events was reduced by $11 \%$ in the first year of treatment, $24 \%$ in the second year, $33 \%$ in years three to five, and by $36 \%$ thereafter $(\mathrm{P}<0.001$ for trend). IHD events were reduced by $20 \%, 31 \%$, and $51 \%$ in trials grouped by LDL cholesterol reduction (means $0.5 \mathrm{mmol} / \mathrm{l}, 1.0 \mathrm{mmol} / \mathrm{l}$, and $1.6 \mathrm{mmol} / \mathrm{l}$ ) after results from first two years of treatment were excluded $(\mathrm{P}<0.001$ for trend). After several years a reduction of $1.8 \mathrm{mmol} / \mathrm{l}$ would reduce IHD events by an estimated $61 \%$. Results from the same 58 trials, corroborated by results from the nine cohort studies, show that lowering LDL cholesterol decreases all stroke by $10 \%$ for a $1 \mathrm{mmol} / \mathrm{l}$ reduction and $17 \%$ for a $1.8 \mathrm{mmol} / \mathrm{l}$ reduction. Estimates allow for the fact that trials tended to recruit people with vascular disease, among whom the effect of LDL cholesterol reduction on stroke is greater because of their higher risk of thromboembolic stroke (rather than haemorrhagic stroke) compared with people in the general population.
\end{abstract}

Conclusions Statins can lower LDL cholesterol concentration by an average of $1.8 \mathrm{mmol} / \mathrm{l}$ which reduces the risk of IHD events by about $60 \%$ and stroke by $17 \%$.

\section{Introduction}

Statins are highly effective in lowering serum cholesterol concentrations and preventing ischaemic heart disease (IHD) ${ }^{1-3}$ Three issues remain. We do not know by how much different statins at different doses reduce low density lipoprotein (LDL) cholesterol concentrations. There are indications from previous studies, ${ }^{45}$ but there has been no systematic review of placebo controlled trials. Secondly, the full effect of statins in preventing IHD events has not been specified. The 30\% reduction shown in meta-analyses of major randomised trials ${ }^{1-3}$ underestimates the full effect because IHD events in the first two years (before the full effect of reducing serum LDL cholesterol concentrations is achieved ${ }^{6}$ ) were not censored, many trials used less effective statins, and trials were affected by non-adherence to the allocated regimen (those on statins not taking them and those on placebo taking statins). Thirdly, there is a paradox in that metaanalyses of randomised trials showed that statins reduced the incidence of strokes by about $30 \%,{ }^{7-10}$ but cohort studies showed no association between serum cholesterol concentrations and stroke. ${ }^{11}$

\section{Methods}

We carried out three analyses. The first was a meta-analysis of 164 short term (typically a few weeks) randomised placebo controlled trials of six statins (atorvastatin, fluvastatin, lovastatin, pravastatin, simvastatin, and rosuvastatin (recently marketed)), used in fixed dose. ${ }^{\mathrm{w} 1 \mathrm{-w} 164}$ The meta-analysis examined the efficacy of reducing total and LDL cholesterol by dose and pretreatment serum cholesterol concentrations. The second meta-analysis was of 58 randomised trials (including eight of the above 164 trials) of reducing serum cholesterol concentration by any means and IHD events to estimate the reduction in risk by LDL cholesterol reduction and duration of treatment. This updates our 1994 analyses. ${ }^{6}{ }^{12}$ In the third analysis we examined data from nine cohort studies and the 58
Department of

Environmental and Preventive

Medicine, Wolfson

Institute of

Preventive

Medicine, Barts and the London, Queen Mary's School of

Medicine and

Dentistry, University of London, London EC1M 6BQ

M R Law

professor

NJ Wald

professor

A R Rudnicka

lecturer

Correspondence to: M R Law

m.r.law@qmul.ac.uk

bmj.com 2003;326:1423

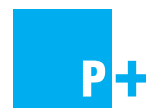

A full list of references and two extra tables can be found on bmi.com 
Table 1 Details of 164 randomised placebo controlled trials of statins and serum cholesterol reduction. Figures are means ( $90 \%$ range) unless stated otherwise

\begin{tabular}{|c|c|c|}
\hline & Treatment & Placebo \\
\hline \multicolumn{3}{|c|}{ No of treatment groups (participants) in trials of: } \\
\hline Atorvastatin ${ }^{w 1-w 15}$ & $24(2217)$ & $15^{\star}(2051)$ \\
\hline Fluvastatin $^{\text {w16-w38 }}$ & $31(3054)$ & $23(1675)$ \\
\hline Lovastatin"14 w39-w52 & $28(7938)$ & $15(2710)$ \\
\hline Pravastatin ${ }^{\text {w14 w52-w120 }}$ & $85(5474)$ & 70 (4382) \\
\hline Rosuvastatin $^{\text {w2 w121 }}$ & $9(394)$ & $2(161)$ \\
\hline Simvastatinn14 w15 w116-w120 w122-w164 & $72(4906)$ & $50(3933)$ \\
\hline All trials & $249(23983)$ & $164 \dagger(14320)$ \\
\hline No of participants per treatment group & $95(8-396)$ & $87(7-412)$ \\
\hline \multicolumn{3}{|l|}{ No of treatment arms per design: } \\
\hline Crossover & 42 & 33 \\
\hline Parallel groups & 207 & 131 \\
\hline \multicolumn{3}{|c|}{ Serum cholesterol (mmol/l) concentrations before treatment: } \\
\hline Total cholesterol & $6.8(5.6-9.9)$ & $6.7(5.3-9.5)$ \\
\hline LDL cholesterol & $4.8(3.2-7.4)$ & $4.6(3.0-6.8)$ \\
\hline Median duration (weeks) & $8(4-48)$ & $8(4-48)$ \\
\hline Age (years) & $55(43-71)$ & $55(43-70)$ \\
\hline
\end{tabular}

${ }^{*}$ Number of placebo groups is same as number of trials.

†Less than total of five categories because some trials compared two or more statins with same placebo group.

randomised trials to determine the effect of a decrease in LDL cholesterol concentration on thromboembolic, haemorrhagic, fatal, and non-fatal stroke.

164 short term trials of statins and LDL cholesterol reduction

We searched Medline, Cochrane Collaboration, and Web of Science databases (see www.smd.qmul.ac.uk/ wolfson/bpchol for details and webextra for full list of references $\left.{ }^{\mathrm{w1}-\mathrm{w} 164}\right)$. We included all double blind trials, irrespective of participants' age or disease. Participants in most trials were healthy with above average lipid concentrations. In some trials they had high blood
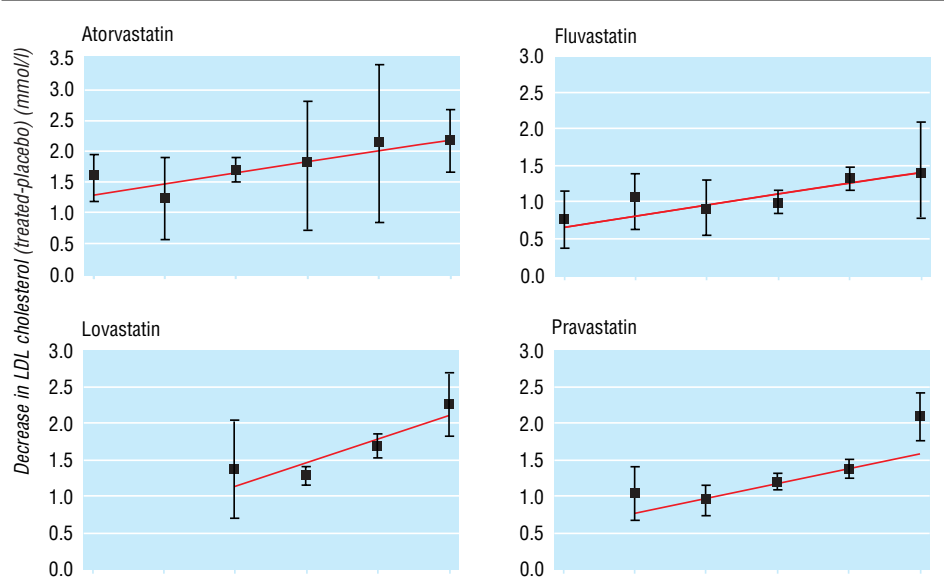

Pravastatin
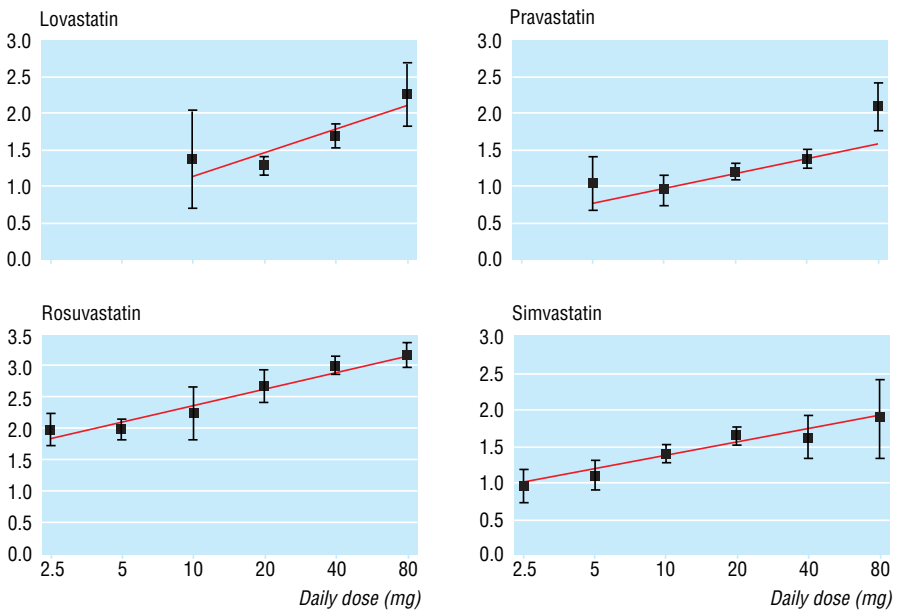

Fig 1 Average reductions in LDL cholesterol concentration (95\% confidence intervals) in the 164 trials according to statin and dose (not standardised to pretreatment serum cholesterol concentration) pressure, diabetes, or IHD. We excluded trials that had no placebo group, lasted less than two weeks, used variable doses (titrating), or used cholesterol lowering drugs in combination, and trials in which the order of treatment and placebo periods in crossover trials was not randomised or patients had chronic renal failure or organ transplantation.

We defined drug efficacy as the reduction in LDL cholesterol concentration for a given dose, expressed as the change in the treated group minus that in the placebo group (in crossover trials end treatment minus end placebo concentration). Methods for calculating standard errors and the statistical analyses are described in the accompanying paper. ${ }^{14}$

\section{8 randomised trials of serum cholesterol} reduction (by any means) and IHD events

We expanded the literature search to include methods of reduction of serum cholesterol concentrations other than statins; 33 more recent trials and 25 reported in $1994^{6}$ met inclusion criteria. We excluded trials in which risk factors other than lipids were changed, LDL cholesterol reduction was $<0.2 \mathrm{mmol} / \mathrm{l}$, fewer than five IHD events were recorded, or there was no untreated control group. We calculated the absolute change in serum LDL cholesterol concentration in the treatment group minus that in the placebo group. In 17 of the 25 earlier trials LDL cholesterol was not measured so we used total serum cholesterol concentration. We defined IHD events as IHD death or non-fatal myocardial infarction, ignoring subsequent events in an individual and excluding "silent" infarcts. In each trial we determined the numbers of IHD events and the changes in LDL cholesterol (adjusted for placebo) separately for years one, two, three to five, and six or more after trial entry. We recorded disease events and the average reduction in LDL cholesterol concentration in all randomised participants regardless of compliance (assuming LDL concentration reverted to baseline when participants left the trial).

We combined the odds ratios (treated/placebo) of disease events, stratified according to duration of scheduled treatment, to yield summary estimates using a random effects model. ${ }^{15}$ After the effects of reduction in LDL cholesterol and duration of treatment were taken into account there was no significant residual heterogeneity. We standardised each trial result to an LDL cholesterol reduction of $1.0 \mathrm{mmol} / \mathrm{l}$ by raising the observed odds ratio to the power of (1.0 divided by the observed LDL cholesterol reduction).

\section{Nine cohort studies and 58 randomised trials of serum cholesterol and stroke}

We identified nine cohort studies of serum cholesterol concentration and stroke that distinguished thromboembolic and haemorrhagic strokes using computed tomography or postmortem findings. We used Medline (1980 to October 2002; key and text words blood cholesterol and [cerebral haemorrhage or intracranial haemorrhages or subarachnoid haemorrhage or cerebral infarction]). We determined the difference in incidence for a difference in LDL cholesterol of 1.0 $\mathrm{mmol} / \mathrm{l}$ adjusted for regression and surrogate dilution bias. ${ }^{12}$ Data on stroke from the 58 randomised trials were combined by using a random effects model. ${ }^{15}$ There was no significant heterogeneity. 
Table 2 Absolute reductions* (mmol/l) (with 95\% confidence intervals) and percentage reductions $\dagger$ in serum LDL cholesterol concentration according to statin and daily dose (summary estimates from 164 randomised placebo controlled trials)

\begin{tabular}{lccccc} 
& \multicolumn{5}{c}{ Daily dose (mg) } \\
\cline { 2 - 6 } Statin & $\mathbf{5}$ & $\mathbf{1 0}$ & $\mathbf{2 0}$ & $\mathbf{4 0}$ & $\mathbf{8 0}$ \\
\hline Atorvastatin & $1.51(1.28$ to 1.74$), 31 \%$ & $1.79(1.62$ to 1.97$), 37 \%$ & $2.07(1.90$ to 2.25$), 43 \%$ & $2.36(2.12$ to 2.59$), 49 \%$ & $2.64(2.31$ to 2.96$), 55 \%$ \\
\hline Fluvastatin & $0.46(0.18$ to 0.75$), 10 \%$ & $0.74(0.55$ to 0.93$), 15 \%$ & $1.02(0.90$ to 1.13$), 21 \%$ & $1.30(1.19$ to 1.41$), 27 \%$ & $1.58(1.40$ to 1.76$), 33 \%$ \\
\hline Lovastatin & - & $1.02(0.71$ to 1.34$), 21 \%$ & $1.40(1.21$ to 1.59$), 29 \%$ & 1.77 (1.60 to 1.94$), 37 \%$ & $2.15(1.86$ to 2.43$), 45 \%$ \\
\hline Pravastatin & $0.73(0.54$ to 0.92$), 15 \%$ & $0.95(0.83$ to 1.07$), 20 \%$ & $1.17(1.10$ to 1.23$), 24 \%$ & 1.38 (1.31 to 1.46$), 29 \%$ & $1.60(1.46$ to 1.74$), 33 \%$ \\
\hline Rosuvastatin & $1.84(1.74$ to 1.94$), 38 \%$ & $2.08(1.98$ to 2.18$), 43 \%$ & $2.32(2.20$ to 2.44$), 48 \%$ & $2.56(2.42$ to 2.70$), 53 \%$ & $2.80(2.63$ to 2.97$), 58 \%$ \\
\hline Simvastatin & $1.08(0.93$ to 1.22$), 23 \%$ & $1.31(1.22$ to 1.40$), 27 \%$ & $1.54(1.46$ to 1.63$), 32 \%$ & 1.78 (1.66 to 1.90$), 37 \%$ & $2.01(1.83$ to 2.19$), 42 \%$ \\
\hline
\end{tabular}

*Absolute reductions are standardised to usual serum LDL cholesterol concentration of $4.8 \mathrm{mmol} / \mathrm{l}$ before treatment (mean concentration in trials).

†Percentage reductions are independent of pretreatment LDL cholesterol concentration; $95 \%$ confidence intervals on percentage reductions can be derived by dividing those on absolute reductions by 4.8 .

Table 3 Reduction in risk (95\% confidence intervals) of ischaemic heart disease events ${ }^{*}$ for $1.0 \mathrm{mmol} / \mathrm{l}$ decrease in serum LDL cholesterol concentration, according to number of years in trial $(58$ trials $)$

\begin{tabular}{ll} 
Year in trial & \% Reduction in risk \\
\hline 1st & $11(4$ to 18$)$ \\
\hline 2nd & $24(17$ to 30$)$ \\
\hline 3rd-5th & $33(28$ to 37$)$ \\
\hline 6th and subsequent & $36(26$ to 45$)$ \\
\hline
\end{tabular}

${ }^{*} \mathrm{HHD}$ death and non-fatal myocardial infarction.

\section{Results}

164 short term trials of statins and LDL cholesterol reduction

Table 1 shows details of the 164 trials. There were about 24000 treated and 14000 placebo participants (individual trial data on can be found on www.smd.qmul.ac.uk/wolfson/bpchol).

Figure 1 shows the dose-response relations for the five statins across the doses tested $(2.5-80 \mathrm{mg} /$ day $)$. The straight lines fit the data well. With simvastatin the linear trend is unconvincing above $20 \mathrm{mg}$ /day, but one study (excluded from our meta-analysis because it had no placebo group) confirmed greater efficacy at higher doses. ${ }^{16}$

Table 2 shows the estimated reductions in LDL cholesterol, according to statin and dose, calculated from the straight lines and standardised to the average pretreatment LDL cholesterol concentration in these trials $(4.8$ $\mathrm{mmol} / \mathrm{l}$; about the average in people having an IHD event). Rosuvastatin $5 \mathrm{mg}$ /day, atorvastatin $10 \mathrm{mg} /$ day, and lovastatin or simvastatin $40 \mathrm{mg}$ /day reduced LDL cholesterol concentrations by about $35 \%$ (1.8 mmol/l), but fluvastatin and pravastatin produced smaller reductions even at the highest doses tested $(80 \mathrm{mg} /$ day $)$. Rosuvastatin $10 \mathrm{mg} /$ day, atorvastatin $20 \mathrm{mg}$ /day, and lovastatin or simvastatin $80 \mathrm{mg}$ /day reduced concentrations by about $45 \%(2.1 \mathrm{mmol} / \mathrm{l})$ and rosuvastatin $80 \mathrm{mg} /$ day by about $60 \%(2.8 \mathrm{mmol} / \mathrm{l})$.

Statins significantly lowered LDL cholesterol from all pretreatment concentrations. The absolute reductions (in $\mathrm{mmol} / \mathrm{l}$ ) were greater in those with higher pretreatment concentrations. The percentage reductions were independent of pretreatment concentrations and therefore more generalisable, but we adopted absolute reductions because the relations with disease events were quantified by using absolute cholesterol reductions. ${ }^{6}$ If the pretreatment concentration was $1 \mathrm{mmol} / \mathrm{l}$ higher $(5.8 \mathrm{mmol} / \mathrm{l})$, LDL cholesterol reduction was on average $0.28 \mathrm{mmol} / \mathrm{l}$ greater. No effect of age was apparent, but there was little variation in average age across trials.
In these 164 trials atorvastatin and rosuvastatin were taken in the morning but the other statins in the evening. In four randomised comparisons in three trials $^{\mathrm{w} 77 \mathrm{w} 137} \mathrm{w} 153$ the average reduction was $0.20 \mathrm{mmol} / \mathrm{l}$ smaller with morning dose than with evening dose (95\% confidence interval $0.05 \mathrm{mmol} / \mathrm{l}$ to $0.44 \mathrm{mmol} / \mathrm{l}$ smaller). Doubling the dose has a similar effect (about $0.20 \mathrm{mmol} / \mathrm{l}$ ) and so would counter this. The greater effect of evening dose arises because of short biological half life (peak cholesterol synthesis occurs at night). Atorvastatin and rosuvastatin have longer half lives and avoid this problem; lipid changes with atorvastatin were similar with morning and evening dose. ${ }^{17}$

The reductions in total and LDL cholesterol concentrations were highly correlated across trials $(r=0.83)$. On average, a reduction in LDL cholesterol of $1.0 \mathrm{mmol} / \mathrm{l}$ was associated with a total cholesterol reduction of $1.20 \mathrm{mmol} / \mathrm{l}(1.10 \mathrm{mmol} / \mathrm{l}$ to $1.31 \mathrm{mmol} /$ l). If only total serum cholesterol reduction were measured, we would expect the absolute reduction in LDL cholesterol to be $17 \%$ less (1.0 minus $1.0 / 1.20)$. The reduction in VLDL cholesterol was on average $10 \%$ of the reduction in LDL cholesterol shown in table 2. Statins increased HDL cholesterol by 0.07 $\mathrm{mmol} / \mathrm{l}(0.06 \mathrm{mmol} / \mathrm{l}$ to $0.08 \mathrm{mmol} / \mathrm{l})$ on average, with no detectable effect of dose.

\section{8 trials of serum cholesterol reduction and IHD events}

These 58 trials included 76359 participants allocated treatment and 71962 controls, with 5440 and 7102 IHD events respectively: $52 \%$ of participants had known vascular disease on entry. See webextra tables A and $\mathrm{B}$ for details.

Table 3 shows the reduction in IHD events by duration of treatment; each trial result is standardised to a reduction in LDL cholesterol of $1.0 \mathrm{mmol} / \mathrm{l}$ (about the average reduction in the trials). In the first year the reduction was $11 \%$, in the second $24 \%$, and in the first and second years combined 13\%. Data from 12 trials with event numbers published for the first two years but not the first and second years separately yielded a similar result. The reduction in the third, fourth, and fifth years combined was 33\%, and the sixth and subsequent years was 36\%. After standardisation for reduction in LDL cholesterol and duration of treatment, risk reduction was similar for fatal and nonfatal IHD events, for different methods of reducing serum cholesterol (fibrates, resins, niacin, statins, or dietary change), and in participants with and without known IHD on entry (showing that the proportional risk reduction model applies regardless of initial risk). 


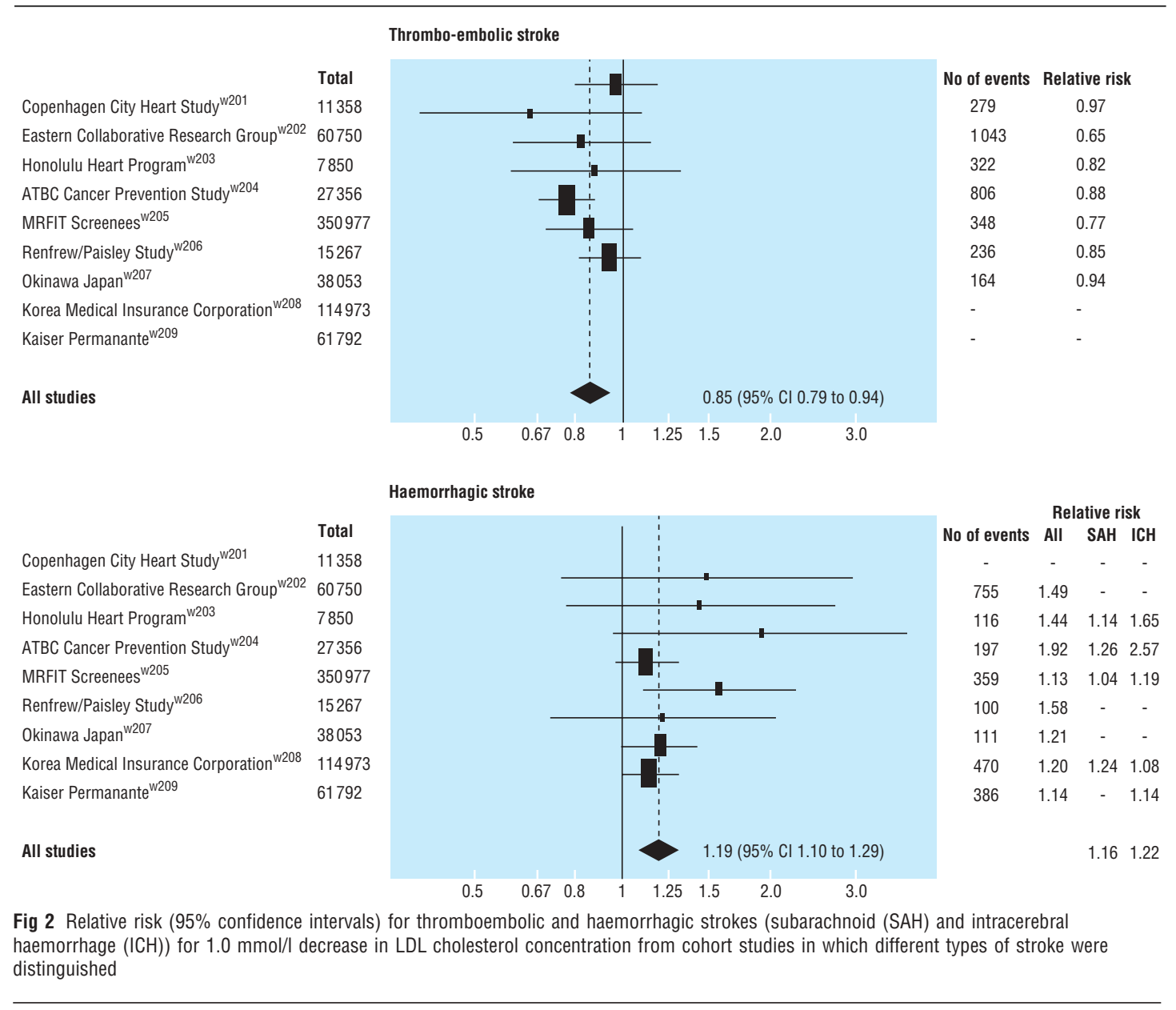

Table 4 shows the combined effect of duration of treatment and reduction in LDL cholesterol directly. The trials tend to cluster into three groups, with mean reductions of LDL cholesterol of $0.5 \mathrm{mmol} / \mathrm{l}(\mathrm{n}=21)$, $1.0 \mathrm{mmol} / \mathrm{l}(\mathrm{n}=24)$, and $1.6 \mathrm{mmol} / \mathrm{l}(\mathrm{n}=5)$. Across these groups, the greater the reduction in LDL cholesterol the greater the reduction in IHD events. With a reduction in LDL cholesterol of around $1.6 \mathrm{mmol} / \mathrm{l}$ the reduction in IHD events after two or more years' treatment was $51 \%$.

\section{Serum cholesterol and stroke}

Nine cohort studies

Figure 2 shows the relative risk of thromboembolic and haemorrhagic stroke for a $1.0 \mathrm{mmol} / \mathrm{l}$ decrease in LDL cholesterol concentration from each of the nine cohort studies that distinguished the two. Overall there was a

Table 4 Reduction (\%) in risk of ischaemic heart disease events (relative odds reduction) in 49 randomised trials* according to number of years in trial at time of event and reduction in LDL cholesterol concentration

\begin{tabular}{|c|c|c|c|c|}
\hline \multirow[b]{2}{*}{ Year in trial } & \multicolumn{3}{|c|}{ LDL cholesterol reduction $(\mathrm{mmol} / \mathrm{l})$} & \multirow{2}{*}{$\begin{array}{l}P \text { value (test fo } \\
\text { trend) }\end{array}$} \\
\hline & $0.2-0.7^{\star}$ & $0.8-1.4 \dagger$ & $\geq 1.5 \neq$ & \\
\hline 1st and 2nd§ & 6 & 19 & 33 & 0.015 \\
\hline 3rd, 4th, and 5th & 19 & 31 & 50 & $<0.001$ \\
\hline 6th and subsequent & 21 & 30 & 52 & 0.026 \\
\hline
\end{tabular}

*21 trials, mean reduction $0.5 \mathrm{mmol} / \mathrm{l}$.

†24 trials, mean reduction $1.0 \mathrm{mmol} / \mathrm{.}$

$\ddagger 5$ trials, mean reduction $1.6 \mathrm{mmol} / \mathrm{l}$.

$\S$ Excludes 8 trials that lasted less than one year.
$15 \%(6 \%$ to $21 \%)$ decrease in thromboembolic stroke $(\mathrm{P}<0.001)$ and a $19 \%(10 \%$ to $29 \%)$ increase in haemorrhagic stroke $(\mathrm{P}<0.001)$. The similar relative risk estimates for subarachnoid (1.16) and intracerebral haemorrhage (1.22) justify combining them as "haemorrhagic stroke."

The opposing effects of thromboembolic and haemorrhagic stroke explain the absence of an association between serum cholesterol and stroke in a metaanalysis of 45 cohort studies. ${ }^{11}$ Death from the two types of stroke cancelled each other because the 45 cohort studies generally recorded only fatal stroke and at age 60 (the average in the studies) about half of fatal strokes are thromboembolic and half are haemorrhagic. $^{18}{ }^{1010-w 214}$ These data also show that at age 60 , $76 \%$ of non-fatal strokes are thromboembolic and $24 \%$ haemorrhagic, and $71 \%$ of all strokes are thromboembolic and 29\% haemorrhagic ${ }^{18}$ (the difference from fatal stroke explained by the greater chance of dying from a haemorrhagic stroke). Application of the change in risk for type of stroke to these percentages yields an expected decrease in non-fatal stroke (per 1 $\mathrm{mmol} / \mathrm{l}$ reduction in LDL cholesterol) of $7 \%$ (a $15 \%$ decrease in $76 \%$ plus a $19 \%$ increase in $24 \%$ ). Similarly the expected decrease for all stroke is $6 \%$.

58 trials of cholesterol reduction by any means and disease events

Fifty six of the 58 trials reported on deaths from stroke (though in 21 trials none occurred) and 40 reported on 
Table 5 Change in risk of stroke (relative odds reduction) for $1.0 \mathrm{mmol} / /$ reduction in $\mathrm{LDL}$ cholesterol in randomised trials and estimates from analysis of cohort studies (fig 2) according to whether trial participants had known vascular disease on entry and whether stroke was thromboembolic or haemorrhagic and fatal or non-fatal

\begin{tabular}{|c|c|c|c|c|}
\hline \multirow[b]{2}{*}{ Category } & \multicolumn{3}{|c|}{ Randomised trials } & \multirow[b]{2}{*}{$\begin{array}{l}\text { Estimated \% change in risk in } \\
\text { cohort studiest }(95 \% \mathrm{CI})\end{array}$} \\
\hline & No of trials & No of events & $\begin{array}{l}\text { Estimated \% change in risk } \\
\qquad(95 \% \mathrm{Cl})\end{array}$ & \\
\hline All stroke & 41 & 3319 & $-20^{*}(-14$ to -26$)$ & - \\
\hline All stroke in people with known vascular disease & $32 \dagger$ & 2311 & $-22^{*}(-28$ to -16$)$ & - \\
\hline All stroke in people without known vascular disease & $7 \dagger$ & 752 & $-6(-22$ to 14$)$ & $-6 \ddagger(-12$ to 1$)$ \\
\hline Thromboembolic stroke & 8 & 1204 & $-28^{*}(-35$ to -20$)$ & $-15^{\star}(-21$ to -6$)$ \\
\hline Haemorrhagic stroke & 8 & 149 & $-3(-35$ to 47$)$ & $19^{*}(10$ to 29$)$ \\
\hline Fatal stroke & 56 & 678 & $-2(-17$ to 16$)$ & $0 \neq \S(-6$ to 6$)$ \\
\hline Non-fatal stroke & 40 & 2519 & $-23^{*}(-29$ to -16$)$ & $-7 \ddagger(-13$ to -1$)$ \\
\hline
\end{tabular}

${ }^{*} \mathrm{P}<0.001$.

†Trials in which there were similar numbers of strokes in patients with and without vascular disease on entry are omitted, except one in which separate numbers were available."w168

fFrom data in figure 2 (taking account where necessary of proportions of non-fatal and all strokes that are thromboembolic and haemorrhagic).

§Meta-analysis of 45 cohort studies ${ }^{11}$ (in which nearly all strokes recorded were fatal) gave similar result $(2,-1$ to 6$)$.

non-fatal strokes (in 10 none occurred) (see webextra tables A and B for details).

Table 5 shows the main results on stroke from randomised trials and the above expected results from the nine cohort studies, standardised to an LDL cholesterol reduction of $1.0 \mathrm{mmol} / \mathrm{l}$. Stroke risk in all the trials was reduced by $20 \%$ on average $(\mathrm{P}<0.001)$, but this varied. In people without known vascular disease the reduction was the same $(-6 \%)$ as that expected from cohort studies, but in people with known vascular disease it was higher $(-22 \% v-6 \% ; \mathrm{P}<0.001)$. This difference probably arises because thromboembolic stroke is common in people with vascular disease so more of their strokes will be thromboembolic. Results from the six randomised trials in people with known vascular disease that distinguished the two types of stroke confirmed this: $91 \%$ of the strokes in the placebo groups were thromboembolic $(700 / 773)$ and $9 \%$ haemorrhagic, whereas in the stroke registry studies in people the same age without known vascular disease $71 \%$ of strokes were thromboembolic and $29 \%$ haemorrhagic. ${ }^{\text {w210-w214 }}$ Reduction in LDL cholesterol concentration prevents thromboembolic but not haemorrhagic strokes, accounting for the greater than expected effect of treatment in people with vascular disease. This also explains the greater than expected reduction in non-fatal stroke in shown table $5(-23 \% v$ $-7 \% ; \quad \mathrm{P}<0.001)$ as most non-fatal strokes are thromboembolic. The absence of a material reduction in fatal stroke in the trials corroborates the cohort study observations.

The $20 \%$ reduction in stroke for a $1.0 \mathrm{mmol} / \mathrm{l}$ reduction in LDL cholesterol concentration is therefore specific to these trial populations in which $80 \%$ of all strokes were in people with known vascular disease. In the general population, stroke registry data indicate that about $25 \%$ of first strokes are in people with known vascular disease. ${ }^{\text {w217-w219 }}$ Therefore a reduction of $1.0 \mathrm{mmol} / \mathrm{l}$ in LDL cholesterol would reduce stroke in the general population by $10 \%(25 \%$ of the $22 \%$ reduction in people with known vascular disease and $75 \%$ of the $6 \%$ reduction in people without known vascular disease, from table 5).

Three large trials showed little or no reduction in incidence of stroke until the second year, as for IHD. ${ }^{\mathrm{w} 165}{ }^{\mathrm{w} 184-\mathrm{w} 187}$ The reductions in all stroke, thromboembolic stroke, and non-fatal stroke shown in table 5 would, therefore, be greater if events occurring in the first 1-2 years were excluded.

\section{Adverse effects}

Forty eight of the 164 trials of statins and LDL cholesterol reported the number of participants with one or more symptoms possibly caused by the drug (1063/14197 allocated to statins and 923/10568 allocated to placebo). Meta-analysis of these data showed no excess risk in people allocated to statins. On average $1 \%$ fewer treated patients than placebo patients reported symptoms $(95 \%$ confidence interval $3 \%$ fewer to $1 \%$ more in treated patients). The prevalence of each of 12 specific symptoms, including muscle pain and various gastrointestinal symptoms, was similar in treated and placebo patients, even for the highest daily dose tested ( $80 \mathrm{mg}$ for all six statins). The upper confidence limits excluded the possibility that statins caused any symptom in more than $2 \%$ of treated patients.

The only known serious adverse effects of statins are rhabdomyolysis and liver failure from hepatitis. The absolute risks are low. In the trials of statins and adverse events, with about 35000 people and 158000 person years of observation in both treated and placebo groups (see webextra table A), rhabdomyolosis was diagnosed (variable criteria) in eight treated and five placebo patients, none with serious illness or death. Raised serum creatine kinase activity $(\geq 10$ times the "upper limit of normal," used to recognise rhabdomyolysis) was reported in 55 treated patients $(0.17 \%)$ and 43 placebo patients $(0.13 \%)$; muscle symptoms were present in 13 and 4 respectively. From the first marketing of statins in 1987 to May 2001 the Food and Drug Administration recorded 42 deaths from rhabdomyolysis attributable to statins (other than cerivastatin, which was not used in these trials) in the United States ${ }^{19}$ a rate of one per 10 million prescriptions dispensed $^{19}$ or (as a prescription is typically for one month's supply ${ }^{20}$ ) about one per million person years of use. There were no cases of liver failure in the trials. Raised alanine aminotransferase activity ( $\geq 3$ times the upper limit of normal, used to recognise hepatitis) was reported in 449 treated $(1.3 \%)$ and 383 placebo patients (1.1\%) (see webextra table A). From 1987 to May 2000 the Food and Drug Administration recorded 30 cases of liver failure attributable to statins, ${ }^{21}$ again about one per million person years of use. 
Table 6 Percentage reduction (95\% confidence interval) in risk of ischaemic heart disease events in randomised trials (excluding first two years of treatment) compared with expected reductions from cohort study data ${ }^{6}$

\begin{tabular}{|c|c|c|c|c|}
\hline \multirow[b]{2}{*}{ Serum cholesterol reduction ( $\mathrm{mmol} / \mathrm{l})$} & \multirow{2}{*}{$\begin{array}{l}\text { Mean reduction } \\
(\mathrm{mmol} / \mathrm{l})\end{array}$} & \multirow{2}{*}{$\begin{array}{l}\text { Total no of } \\
\text { events }\end{array}$} & \multicolumn{2}{|c|}{ Reduction in risk of IHD events } \\
\hline & & & Observed in trial data & Expected from cohort study data* \\
\hline 0.2 & 0.5 & 2311 & 20 (7 to 31$)$ & 23 (20 to 26$)$ \\
\hline 0.8 & 1.0 & 3556 & 32 (27 to 36$)$ & 41 (37 to 45$)$ \\
\hline$\geq 1.5$ & 1.6 & 705 & 51 (42 to 58$)$ & 57 (52 to 61$)$ \\
\hline
\end{tabular}

${ }^{*}$ At age 60 years (average age at which ischaemic heart disease events occurred).

Table 7 Expected \% decrease in incidence of ischaemic heart disease events for specified decreases in serum cholesterol according to age at event, based on 10 largest cohort studies of serum cholesterol and ischaemic heart disease ${ }^{6}$

\begin{tabular}{llllllll} 
& \multicolumn{7}{c}{ LDL cholesterol reduction $(\mathbf{m m o l} / \mathbf{l})^{*}$} \\
\cline { 2 - 8 } Age (years) & $\mathbf{0 . 6}$ & $\mathbf{1 . 0}$ & $\mathbf{1 . 4}$ & $\mathbf{1 . 8}$ & $\mathbf{2 . 2}$ & $\mathbf{2 . 6}$ & $\mathbf{3 . 0}$ \\
\hline 50 & 39 & 56 & 68 & 77 & 84 & 68 & 74 \\
\hline 60 & 27 & 41 & 52 & 61 & 68 & 79 \\
\hline 70 & 20 & 31 & 41 & 49 & 56 & 62 & 67 \\
\hline
\end{tabular}

*Decreases in incidence follow from linear dose-response relation indicating constant proportional change in risk for specified change in cholesterol. Thus at age 60 years relative risk for decrease of $1.0 \mathrm{mmol} / \mathrm{l}$ is 0.59 ( $41 \%$ decrease), therefore $0.59^{1.4}=0.48$ (52\% decrease) for $1.4 \mathrm{mmol} / \mathrm{l}$ decrease.

Concern over hazards from serum cholesterol reduction was resolved by earlier studies. ${ }^{9}{ }^{13}$ Data from the 58 randomised trials of cholesterol reduction and disease events confirm this. The odds ratios (treated/ placebo) for a $1.0 \mathrm{mmol} / \mathrm{l}$ decrease in serum cholesterol were 0.87 ( 0.73 to 1.03 ; 675 deaths) for circulatory diseases other than IHD and stroke, 1.06 (0.96 to $1.16 ; 2293$ deaths) for cancer, 0.94 (0.72 to $1.23 ; 324$ deaths) for injuries and suicide, and 0.88 ( 0.78 to 1.01 ; 1363 deaths) for diseases other than circulatory diseases and cancer.

\section{Discussion}

Randomised trials show directly that a reduction in LDL cholesterol of $1.6 \mathrm{mmol} / \mathrm{l}$ halves the risk of IHD events after two years and that this reduction can be achieved with low doses of some statins (for example, simvastatin $20 \mathrm{mg} /$ day, table 2). Certain statins achieve larger reductions (for example, $2.6 \mathrm{mmol} / \mathrm{l}$ with atorvastatin $80 \mathrm{mg} /$ day and $2.8 \mathrm{mmol} / \mathrm{l}$ with rosuvastatin 80 $\mathrm{mg} /$ day), which would lead to greater reductions in IHD events, but the corresponding risk reduction cannot be quantified directly from randomised trials as no trial achieved so large a reduction. This can be determined from cohort studies of cholesterol and IHD if cohort studies accurately predicted trial results.

Table 6 shows the reduction in IHD events in the trials after the exclusion of data from the first two years and those expected from cohort study results. ${ }^{6}$ Observed trial and expected cohort study results are close, showing that cohort study data can be used to predict risk reduction from lowering LDL cholesterol. The similarity between trial and cohort study data, and the similar reductions in risk (given cholesterol reduction) with different methods of lowering cholesterol, indicate that the reduction in risk is directly attributable to cholesterol reduction; there is no need to invoke other actions of drugs.

Table 7 shows the reductions in IHD events at different ages predicted from the cohort studies. ${ }^{6}$ At age 60 years a $2.2 \mathrm{mmol} / \mathrm{l}$ reduction in serum LDL cholesterol concentration (attainable by using atorvastatin $40 \mathrm{mg}$ /day, lovastatin $80 \mathrm{mg}$ /day, or rosuvastatin $20 \mathrm{mg}$ /day) would reduce the risk of IHD by nearly $70 \%$. However, adverse effects are also dose related, ${ }^{19}$ and rosuvastatin is relatively untested. As moderate doses of statins substantially reduce the risk of IHD events it may be prudent to select commonly used doses of the older drugs for general use. This would also be cheaper, as simvastatin comes off patent in 2003 and lovastatin is already off patent. At doses of 40 $\mathrm{mg}$ /day these drugs lower LDL cholesterol by 1.8 $\mathrm{mmol} / \mathrm{l}$, which can reduce IHD events at age 60 years by $61 \%(51 \%$ to $71 \%)$. This is about double the currently recognised preventive effect of $30 \% .^{1-3}$

\section{Reasons for underestimation of effect on IHD}

Why are the current estimates of effect so low? Firstly, five of the seven largest statin trials used pravastatin, which is relatively less effective (table 2). Secondly, risk falls relatively little within the first two years, and inclusion of these early events underestimates the preventive effect. Thirdly, a particular problem for the statin trials was the extent to which the intention to treat analysis underestimated the true preventive pharmacological effect because of non-adherence to the protocol (treated patients not taking their tablets and placebo patients taking statins). This last problem was partially overcome by relating the reduction in disease events to the average LDL cholesterol reduction in all randomised participants (treated and placebo). In this way non-adherence to the protocol was reflected in both a smaller than expected difference in LDL cholesterol concentration and a smaller than expected difference in the number of IHD events between the two groups. While this yields an accurate estimate of the risk reduction for the observed difference in LDL cholesterol it underestimates the effect of a given dose of the statin.

\section{Effects of LDL cholesterol reduction on stroke}

The estimated overall reduction in stroke of 10\% (relative risk 0.90 ) for a $1.0 \mathrm{mmol} / \mathrm{l}$ reduction in LDL cholesterol is equivalent to a $17 \%(9 \%$ to $25 \%)$ reduction in stroke for a $1.8 \mathrm{mmol} / \mathrm{l}$ reduction LDL cholesterol, readily achievable with a statin $\left(\right.$ as $0.90^{1.8 / 1}=$ relative risk of 0.83 ). In people with existing vascular disease the reduction is $36 \%\left(0.78^{1.8}=0.64\right)$ The interpretation of the cohort study result showing a higher incidence of haemorrhagic stroke for a lower LDL cholesterol concentration is uncertain. Too few haemorrhagic strokes were identified in the randomised trials to resolve the 


\section{What is already known on this topic}

Statins lower LDL cholesterol, but the size of the reduction according to statin and dose is uncertain

Statins prevent heart disease, but meta-analyses of randomised trials have underestimated their effect

The effect of statins on risk of stroke is uncertain

\section{What this study adds}

Simvastatin $40 \mathrm{mg} /$ day, lovastatin $40 \mathrm{mg} /$ day, and atorvastatin $10 \mathrm{mg}$ /day lower LDL cholesterol by about $37 \%$ from all pretreatment concentrations

These interventions reduce the risk of ischaemic heart disease events at age 60 by an estimated $61 \%$ in the long term, with little reduction in the first year but a $51 \%$ reduction by the third year

The interventions reduce the overall risk of stroke by $17 \%$, preventing thromboembolic but not haemorrhagic stroke

uncertainty. An increased risk cannot be excluded, but this should not preclude the use of statins in the prevention of cardiovascular disease.

\section{Conclusions}

Statins can reduce IHD events by an estimated $61 \%$. They reduce stroke by $17 \%$, preventing non-fatal strokes with little effect on the risk of fatal stroke. Any possible excess of haemorrhagic stroke is greatly outweighed by the protective effect against IHD events and thromboembolic stroke.

We thank the following authors for unpublished data from trials: V Athyros (GREACE ${ }^{\text {w173 }}$ ), M Bortolini and P Serruys $\left(\mathrm{LIPS}^{\mathrm{w17}}{ }^{\mathrm{17}}\right)$, A Tonkin and A Kirby (LIPID $\left.{ }^{\mathrm{w183}}\right)$, T Pedersen and T Cook $\left(4 S^{\mathrm{w1} 156}\right)$, and G Steiner (DAIS $\left.{ }^{\mathrm{w} 200}\right)$, as well as the authors acknowledged in our earlier paper. ${ }^{6}$ We also thank Leo Kinlen for his comments on the manuscript.

Contributors: MRL, NJW, and ARR wrote the paper. MRL and ARR abstracted the data. ARR carried out the statistical analyses. All authors interpreted the results. MRL is guarantor. Funding: ARR was supported by an NHS research and development programme award. The guarantor accepts full responsibility for the conduct of the study, had access to the data, and controlled the decision to publish.
Competing interests: NW and ML have filed a patent application on the formula of a combined pill to simultaneously reduce four cardiovascular risk factors.

1 LaRosa JC, He J, Vupputuri S. Effect of statins on risk of coronary disease. JAMA 1999;282:2340-6.

2 Pignone M, Phillips C, Mulrow C. Use of lipid lowering drugs for primary prevention of coronary heart disease: meta-analysis of randomised trials. BMJ 2000;321:1-5.

3 Gould AL, Rossouw JE, Santanello NC, Hevse JF, Furberg CD. Cholesterol reduction yields clinical benefit. Impact on statin trials. CircuCholesterol reduction y
lation 1998;97:946-52.

4 Jones P, Kafonek S, Laurora I, Hunninghake D. Comparative dose efficacy study of atorvastatin versus simvastatin, pravastatin, lovastatin, and fluvastatin in patients with hypercholesterolemia (the CURVES Study). Am J Cardiol 1998;81:582-7.

5 Schectman G, Hiatt J. Dose-response characteristics of cholesterollowering drug therapies: implications for treatment. Ann Intern Med 1996;125:990-1000.

6 Law MR, Wald NJ, Thompson SG. By how much and how quickly does reduction in serum cholesterol concentration lower risk of ischaemic heart disease? BMJ 1994;308:367-72.

7 Crouse JR, Byington RP, Furberg CD. HMG-CoA reductase inhibitor Crouse JR, Byington RP, Furberg CD. HMG-CoA reductase inhibitor
therapy and stroke risk reduction: an analysis of clinical trials data. therapy and stroke risk redu

8 Blauw GJ, Lagaay AM, Smelt AHM, Westendorp RGJ. A meta-analysis of randomised, placebo controlled, double-blind trials with HMG-CoA reductase inhibitors. Stroke 1997;28:946-50.

9 Hebert PR, Gaziano JM, Chan KS, Hennekens CH. Cholesterol lowering with statin drugs, risk of stroke, and total mortality. JAMA 1997;278:31321.

10 Bucher HC, Griffith LE, Guyatt GH. Effect of HMGcoA reductase inhibitors on stroke. Ann Intern Med 1998;128:89-95.

11 Prospective Studies Collaboration. Cholesterol, diastolic blood pressure and stroke: 13000 strokes in 450000 people in 45 prospective cohorts. and stroke: 13000 strokes
Lancet 1995;346:1647-53.

12 Law MR, Wald NH, Wu T, Hackshaw A, Bailey A. Systematic 2 Law MR, Wald NH, Wu T, Hackshaw A, Bailey A. Systematic
underestimation of association between serum cholesterol concentration and ischaemic heart disease in observational studies: data from the BUPA study. BMJ 1994;308:363-6.

13 Law MR, Thompson SG, Wald NJ. Assessing possible hazards of reducing serum cholesterol. BMJ 1994;308:373-9.

14 Law MR, Wald NJ, Morris JK, Jordan RE. Value of low dose combination treatment with blood pressure lowering drugs: analysis of 354 randomised trials. BMJ 2003; to go in same issue

15 DerSimonian R, Laird N. Meta-analysis in clinical trials. Control Clin Trials 1986;7:177-88.

16 Ose L, Davidson MH, Stein EA, Kastelein JJP, Scott RS, Hunninghake DB, et al. Lipid-altering efficacy and safety of simvastatin $80 \mathrm{mg} /$ day: long-term experience in a large group of patients with hypercholesterolemia. Clin Cardiol 2000;23:39-46.

17 Cilla DD, Gibson DM, Whitfield LR, Sedman AJ. Pharmacodynamic effects and pharmacokinetics of atorvastatin after administration to normocholesterolemic subjects in the morning and evening. J Clin Pharmacol 1996;36:604-9.

18 Sudlow CL, Warlow CP. Comparable studies of the incidence of stroke and its pathological types: results from an international collaboration. International Stroke Incidence Collaboration. Stroke 1997;28:491-9.

19 Staffa JA, Chang J, Green L. Cerivastatin and reports of fatal rhabdomyolysis. N Engl J Med 2002;346:539-40.

20 Wysowski DK, Kennedy DL, Gross TP. Prescribed use of cholesterollowering drugs in the United States, 1978 through 1988. JAMA 1990;263:2185-8.

21 Food and Drug Administration, Center for Drug Evaluation and Research. Statins and hepatotoxicity. www.fda.gov/ohrms/dockets/ac/ 00/backgrd/3622b2b_safety_review.pdf (accessed 8 Apr 2003).

(Accepted 8 April 2003) 